\title{
PROPHYLACTIC MILRINONE MAY DECREASE THE INCIDENCE OF MYOCARDIAL ISCHEMIA DURING OPCAB SURGERY
}

\author{
AUTHORS: H. S. Joo MD, V. N. Naik MD, C. D. Mazer MD; \\ AFFILIATION: Dept of Anesthesia, St. Michael's Hospital, University of Toronto, ON, \\ Canada.
}

INTRODUCTION: Off-pump coronary artery bypass (OPCAB) surgery is associated with decrease in cardiac output and myocardial ischemia, especially during coronary artery clamping and cardiac manipulation. Milrinone, a type 3 phospodiesterase inhibitor, is a positive inotropes with coronary vasodilating properties. We hypothesized that the addition milrinone would increase cardiac output and decrease the incidence of myocardial ischemia during OPCAB surgery.

METHODS: In this double blinded randomized controlled study, adult patients of all ages with grade 1-4 LV's scheduled for OPCAB surgery were prospectively enrolled. After pericardial incision, patients in the milrinone group received a loading dose of 20 $\mathrm{ug} / \mathrm{kg}$ over 20 minutes followed by maintenance of $0.375 \mathrm{ug} / \mathrm{kg}$ and patients in the control group received the same volume of saline as a placebo. Blood pressure was maintained with norepinephrine. Hemodynamic measurements were taken at baseline, 1, 6 and 11 minutes after vessel occlusion. Continuous 5 lead ECG was used for ST analysis. Study infusions were terminated at pericardial closing.

RESULTS: In this ongoing study, 19 patients were enrolled in the milrinone group and 21 patients in the placebo group. Patient demographics were similar. Mean arterial and mean pulmonary artery pressures were similar in both groups. Cardiac index was higher in the milrinone versus control group and maintained during coronary artery cross clamping when compared to baseline $(\mathrm{p}<0.05)$. The incidence of myocardial ischemia was $16 \%$ in the milrinone group and $43 \%$ in the placebo group $(\mathrm{p}=0.09)$. Two patients in each group met enzymatic criteria for myocardial infarction. The incidence of adverse events such as conversion to pump bypass, CVA and death were low and similar in both groups.

DISCUSSION: Milrinone helps maintain cardiac output during cardiac manipulations while the coronary arteries are clamped. Maintenance of cardiac output along with coronary vasodilation may be the mechanism for decreasing the incidence of myocardial ischemia. If the results of this ongoing study continues to favor the decrease in ischemia with milrinone, prophylactic usage of milrinone for OPCAB surgery should be considered. 